\title{
Research on Information Management System of Power Grid Communication Resource
}

\author{
DongdongCai $^{\mathrm{a}}$, Yawei Ding ${ }^{\mathrm{b}}$, YuguiNian, Yongchao Wang \\ State Grid Henan Electric Power Company Luoyang Power Supply Company, Luoyang 471900, \\ China \\ alysc12@126.com, 'b1015903240@qq.com
}

Keywords:Power system communication, resource management, circuit management, scheduling circuitry.

\begin{abstract}
The rapid development of power system communication enables Power Communication network communication devices become more complex, more and more communication lines, which give the communication resource management, especially resource management circuit has posed a serious challenge. This article also power system communication circuit resource management conducted in-depth analysis, and on the basis of standardized circuit application processes through software automation for circuit resource management and scheduling.
\end{abstract}

\section{Introduction}

After decades of development, power communication network has become the communication diverse, well-structured and functional modern communications network, widely used in dispatching telephone system, protection and other aspects of remote power, became the three pillars of power system one. With the rapid development of power communication system, a substantial increase in the number of transmission lines, transmission system capacity increasingly large, while the data network nodes increases, an increasing network bandwidth, making network maintenance and data management becomes very large, resulting in increased network management, the difficulty and complexity of the circuit scheduled work, if the traditional network management methods, will inevitably lead to inefficiency, and error prone.

Communication resource management professional resources involved in large and complex, including line resources and equipment resources, intelligence resources and non-intelligent resources, physical resources and logical resources in the implementation must also consider the relationship with other operations support systems and network management systems. Reasonable planning, design and implementation of the underlying network on the basis of how to provide a wide range of quality and reliable services for the power system, the power to be placed in front of the communications sector is an important issue. The establishment of an integrated, efficient power system communication resource management system is to solve this problem is an important foundation engineering, theoretical significance and application value is very important.

The rapid development of electric power system communication, power system communication resource management has become increasingly complex in foreign countries; the management of communication resources has been a certain amount of development, especially in combination with the existing network management system, gives full play to the communication resources the management role. But in China, the management of communication resources of power or in the manual stage, particularly the management of the circuit resources, still in the way of the use of the card, with the increase in power communication backbone network, this management has been unable to meet the electricity system communication requirements.

\section{Overview of Communication Resource Management}

Communication resource management is an important component of management automation. In recent years, with the development of modern communication technology, constantly updated equipment, communications security has been greatly improved, but due to the coexistence of old and 
new equipment, means of communication range communication resource management system still used in the traditional way, management is difficult, efficiency low, to adapt to the development of communication technology and equipment as soon as possible, we must establish intelligent communications management system, data center overall planning, establishing communication resource management system multi-tier distributed architecture, so that senior management can see the big picture, effective communication means and methods according to the situation and decision support communication resource information transfer information. When the communication system has been damaged can set up emergency communications systems in a short time, in order to meet the urgent need to communicate. While the communications sector can grasp the communication network nodes running status and other information, according to the decision-making information for planning, decision-making and transformation of existing communication systems, determine the optimal communication network structure and configuration, improve the utilization of communication resources.

Communication resource management in the power system, since departments and personnel involved more, large volumes of data and scattered, tedious, there is loss of circuit data duplication, errors, etc., it requires a lot of staff time to manually send a fax, for each kind of business data collection, aggregation and analysis, workload, and the possibility of error is large.

\section{The Function ofInformation Management System of Power Grid Communications Resources}

Power communication resource management system is the actual demand for electric power system communication network resource management proposed a resource management system. It enables circuit resource management, business acceptance, communications station, line management and network device management. Modern electric power communication resource management should be based on geographic information systems technology to build the electric power system communication resources and geographic information systems together, using graphics to visualize the equipment line and indicated resource location information, to maximize the working environment to create illustrations. You can on the basis of geographic information on the city, on a variety of power switching equipment communication system, a variety of cable equipment, line transmission equipment, patch panels, equipment, detailed and accurate to configure, modify, query and maintain, and provide statistical analysis data do not form. Meanwhile, the management of electric power communication resources should achieve easy, accurate and timely geographic maps and equipment distribution lines to be updated to reflect the latest information on network resources, visualization of managed network communications resources. To achieve the sound management of the power system communication resource, design management system must have the following basic functions:

(1) Electrical power communication resource management requirements solve the transmission circuit automatic scheduling and core scheduling problem, implement automated data management. Systems based on digital map system to graphically quickly realize circuit scheduling: Just enter the site information, site equipment information, device port information, channel organizing information, you can implement the circuit scheduling; just enter the site information, the cable segment information, you can achieve core scheduling. The system supports connected to the ports and connectors Cable core information within the entry station, easy to connect the management station. System naming is convention compliant, easy to upgrade and data migration system.

(2) Facilitate the realization of network data input and output and conversion: provides flexible data management, user-friendly network management information needs. Enables automated management of the transmission network all the information, all the information can be provided in Excel format export, to facilitate conversion format printing and mailing.

(3) Intuitive comprehensive resource information management: the system will connect information for all transmission equipment, port and port cable core information, in accordance with the distribution of relations between the station associations. Data entry and inquiry, there are basically three ways: based on pattern according to pattern objects directly query and enter a pattern 
associated object benevolence single attribute case), such as sites, cable segment, core, microwave segment, channel, circuit ; associated graphics object data according to a list of ways to query and enter a graphics object associated benevolence many cases the property) based on patterns, such as equipment, port and connection; relationship based on the transmission system, according to the site name and then query and associatively Enterquickly check the transmission system related equipment), to achieve a transmission device port information query. Specific way: select the type of the transmission system, transmission filter system, input associated site, further filtration equipment, in the election apparatus, the display device ports and port occupancy information. PDH device queries this way is very necessary.

(4) Data entry control framework: The system design should take full account of the communication resource data compilation and entry of complexity, according to the information the degree of importance to distinguish between input methods. Data entry more difficult, try to provide a variety of input methods for users to choose, there are database mode, a picture mode. Information such as cable openings, cable connector system provides data entry way table, but also provides fiber optic cable connecting the image input mode, form complex logic data entry mode, input is complicated, but also an accurate description; image mode entry is simple, but can only play a reference effect.

(5) Data and easy to implement build cycle: According to information on the degree of importance of the information itself, classification, target information, support information and supplementary information. Target information is reflected in the information systems function, support information is objective information as support for information, support information edge information. Under the general management system design principles, the principle of information into supporting information should be entered, and then enter the target information. In general, however, support more complex information and data, grounding is poor, the target information to prepare more fully, relatively brief. If the traditional way, will inevitably result in system data entry difficult, the system opened cycle lengthened, poor usability. We use as much as possible to the way information is entered directly target automatically creates a "virtual support information" does not affect the basic functionality of the system. After the actual support information creation, automatic and '• Virtual support information "related, expand the system functions. For example, some systems is to first build roads and pipelines bar, and then on the road and pipeline lever arm on the cable, the cable core open device, then create ports and channels in order to achieve the circuit information input and scheduling so that the system high precision digital map, pole road and pipeline data is difficult to obtain, nor in fact is the target information so that the system is difficult practical. Our system uses the opposite way, first establish the device logic to achieve circuit scheduling, physical relationship between devices through the "virtual core" connection. After the cable information input, you can achieve a core scheduling, then in the "actual situation of the core correlation between information "to establish a solid connection between the devices. At first, the system is able to complete the most important function, as the deepening of information into the system function more perfect.

(6) The flexibility to control the scale of investment in the system: management system provides a digital map platform, mainly from the role of data processing, the precision with precision of digital maps. Considering the complexity of the digital map production system is not necessary to provide a digital map production platform to reduce software complexity. Map Maker via a dedicated platform

completed by professionals. System platform for network equipment is less demanding, easy to reduce the hardware investment.

\section{Summary}

With the rapid development of power systems for communication, communication devices have become increasingly complex, the subject of automation in the management of system resources, power communication made a good attempt. The purpose communication resource management system is to build a platform for centralized management of network resources to communicate information, and by building a resource management system, network resources to communicate a unified naming convention to establish a scientific business processes, play the level of service to 
enhance the entire enterprise and the role of management level. A communication resource management system must be able to provide an end to end physical and logical perspective and the latest services, shielding the differences of each device manufacturers, smart devices and non-intelligent devices focused on the same software platform, the user the business center offers all related information services.

\section{References}

[1]Peng G, Li H, Li Y. Research and Application of Jinan Electric Power Communication Network Topology Resource Management Information System[J]. Shandong Electric Power, 2011.

[2]Yang J, Wang Y, Zhang X. Technology and Application Research of Power Communication Resources Management System in CSG[J]. Southern Power System Technology, 2009.

[3]Wang S W, Li X, Wang C Y, et al. Research on Annual Operation Mode and Intelligence Aided Design System of Power Communication[J]. Applied Mechanics \& Materials, 2014, 556-562.

[4]Han B Q. Research on graphic information management system of electric communication network[J]. Ningxia Electric Power, 2005.

[5]Cao J. Research on Relative Problem of Communication Network Resource Management System[J]. Telecom Engineering Techniques \& Standardization, 2003.

[6]Zhao G J, Pan B, Yan S, et al. The Intelligent Rural Power Grid Management System Based on Multi-system Fusion[J]. Electric Power Information \& Communication Technology, 2014. 